\title{
A numerical study of Coulomb interaction effects on 2D hopping transport
}

\author{
Yusuf A Kinkhabwala, Viktor A Sverdlov and Konstantin K Likharev \\ Department of Physics and Astronomy, Stony Brook University, Stony Brook, \\ NY 11794-3800, USA
}

Received 25 July 2005, in final form 12 October 2005

Published 27 January 2006

Online at stacks.iop.org/JPhysCM/18/2013

\begin{abstract}
We have extended our supercomputer-enabled Monte Carlo simulations of hopping transport in completely disordered 2D conductors to the case of substantial electron-electron Coulomb interaction. Such interaction may not only suppress the average value of hopping current, but also affect its fluctuations rather substantially. In particular, the spectral density $S_{I}(f)$ of current fluctuations exhibits, at sufficiently low frequencies, a $1 / f$-like increase which approximately follows the Hooge scaling, even at vanishing temperature. At higher $f$, there is a crossover to a broad range of frequencies in which $S_{I}(f)$ is nearly constant, hence allowing characterization of the current noise by the effective Fano factor $F \equiv S_{I}(f) / 2 e\langle I\rangle$. For sufficiently large conductor samples and low temperatures, the Fano factor is suppressed below the Schottky value $(F=1)$, scaling with the length $L$ of the conductor as $F=\left(L_{\mathrm{c}} / L\right)^{\alpha}$. The exponent $\alpha$ is significantly affected by the Coulomb interaction effects, changing from $\alpha=0.76 \pm 0.08$ when such effects are negligible to virtually unity when they are substantial. The scaling parameter $L_{\mathrm{c}}$, interpreted as the average percolation cluster length along the electric field direction, scales as $L_{\mathrm{c}} \propto E^{-(0.98 \pm 0.08)}$ when Coulomb interaction effects are negligible and $L_{\mathrm{c}} \propto E^{-(1.26 \pm 0.15)}$ when such effects are substantial, in good agreement with estimates based on the theory of directed percolation.
\end{abstract}

\section{Introduction}

The hopping transport of quasi-localized electrons in disordered conductors and semiconductors has been studied for many years; for comprehensive reviews, see [1-3]. The more recent observation $[4,5]$ that hopping transport may implement the quasi-continuous ('sub-electron') charge transfer, hence providing a possible solution to the random background charge problem in single-electronics [6], has renewed interest in this phenomenon, with an emphasis on the shot noise of the hopping current [7-10]. The objective of this paper is to present the results of an extension of our previous numerical studies of $2 \mathrm{D}$ hopping $[9,10]$ 
to the case of substantial Coulomb interaction of hopping electrons. Just as in the case of negligible interaction [10], the use of advanced algorithms of spectral density calculation [11] and modern supercomputer facilities has allowed us not only to obtain more complete and exact results for average characteristics of hopping transport (including the dependence of the DC current on temperature and electric field), but also to calculate the spectral density of current fluctuations at low temperatures.

In order to explain our new findings, we have to start with a brief summary of the basic prior results.

\subsection{Coulomb gap}

Most theoretical discussions of the Coulomb interaction effects on hopping are based on the notion of the Coulomb gap in the electron energy spectrum. Generally speaking, substantial Coulomb interaction makes the single-particle energy meaningless. However, the introduction [2] of the effective single-particle energy $\varepsilon$, which includes the contribution from the Coulomb interaction with other electrons, immediately leads to a 'soft' gap in the singleparticle density of states $v(\varepsilon)$ at $\varepsilon \approx \mu$, where $\mu$ is the Fermi level. In the case of 2D conductors with the $3 \mathrm{D}$ Coulomb interaction law, which is the focus of our current work, simple arguments $[2,3]$ yield

$$
v(\varepsilon)=c \frac{\kappa^{2}}{e^{4}}|\varepsilon-\mu|,
$$

where $e$ is electron charge, $\kappa$ is the dielectric constant of the insulating environment and $c$ is a dimensionless constant. Equation (1) is valid only when the $2 \mathrm{D}$ density of states $v(\varepsilon)$ is much smaller than the 'seed' density of states $\nu_{0}$; for larger $\varepsilon$ there is a continuous crossover to $v_{0}$. The effective width $\Delta$ of the Coulomb gap can be estimated from the natural condition $v(\Delta)=v_{0}$, resulting in

$$
\Delta=\frac{e^{4} v_{0}}{c \kappa^{2}}
$$

A self-consistent approach [3] allows a more rigorous evaluation of the Coulomb gap width, giving $c=2 / \pi$.

\subsection{DC transport characteristics}

At low applied electric fields $E$, the average current $\langle I\rangle$ is a linear function of $E$, i.e. the 2D ('sheet') DC conductivity $\sigma(T, E, \chi) \equiv\langle I\rangle / E W$ (where $W$ is the width of the conductor) is independent of $E$. For not very high temperatures $\left(T \ll T_{0}\right.$, where $k_{\mathrm{B}} T_{0} \equiv 1 / \nu_{0} a^{2}$ and $a$ is the localization radius), the ratio $\sigma / \sigma_{0}$ (where $\sigma_{0}$ is a constant characterizing the sample) depends only on two dimensionless parameters: the ratio $T / T_{0}$ and parameter $\chi \equiv e^{2} \nu_{0} a / \kappa$ characterizing the Coulomb interaction strength. The relation between these two parameters determines two possible variable-range hopping transport regimes.

If the Coulomb interaction is weak $\left(\chi^{3} \ll T / T_{0}\right)$, the average length $r(T, E, \chi)$ of the so-called 'critical hops', which connect percolation clusters and hence determine the current, may be found from the Mott theory [1-3]:

$$
r(T, 0,0) \propto\left(\frac{T_{0}}{T}\right)^{1 / 3} a .
$$

In this case the conductivity is [1-3]

$$
\frac{\sigma}{\sigma_{0}} \approx A(T, 0,0) \exp \left[-\left(B(T, 0,0) \frac{T_{0}}{T}\right)^{1 / 3}\right],
$$


where $A(T, E, \chi)$ is a dimensionless, model-dependent, slow function of its arguments, while $B(T, E, \chi)$ is usually treated as a constant, but in general may be also a weakly dependent function of its arguments.

On the other hand, if the Coulomb interaction is strong $\left(\chi^{3} \gg T / T_{0}\right)$, the critical hops are longer [2, 3]:

$$
r(T, 0, \chi) \propto\left(\frac{\chi T_{0}}{T}\right)^{1 / 2} a,
$$

and the DC conductivity is suppressed [2,3]:

$$
\frac{\sigma}{\sigma_{0}} \approx A(T, 0, \chi) \exp \left[-\left(B(T, 0, \chi) \frac{\chi T_{0}}{T}\right)^{1 / 2}\right] .
$$

In the case of relatively high electric fields $\left(E \gg E_{T}\right.$, where $\left.E_{T} \equiv k_{\mathrm{B}} T / e a\right)$, the DC current is a highly nonlinear (exponential) function of the applied electric field $E$. If the field is not extremely high $\left(E \ll E_{0} \equiv 1 / e v_{0} a^{3}\right)$, i.e. in the variable-range hopping domain, we can again distinguish two different transport regimes.

If the Coulomb interaction is weak $\left(\chi^{3} \ll E / E_{0}\right)$, one can neglect the effects of Coulomb interaction to evaluate the critical hop length

$$
r(0, E, 0) \propto\left(\frac{E_{0}}{E}\right)^{1 / 3} a .
$$

In this case, the DC conductivity is [12-16]

$$
\frac{\sigma}{\sigma_{0}} \approx A(0, E, 0) \exp \left[-\left(B(0, E, 0) \frac{E_{0}}{E}\right)^{1 / 3}\right] \text {. }
$$

In the opposite limit $\left(\chi^{3} \gg E / E_{0}\right)$,

$$
r(0, E, \chi) \propto\left(\frac{\chi E_{0}}{E}\right)^{1 / 2} a
$$

and the DC conductivity is lower [15]:

$$
\frac{\sigma}{\sigma_{0}} \approx A(0, E, \chi) \exp \left[-\left(B(0, E, \chi) \frac{\chi E_{0}}{E}\right)^{1 / 2}\right] \text {. }
$$

\subsection{Current fluctuations}

At low temperatures ${ }^{1}$, the dynamical fluctuations of the current flowing through a mesoscopic system are more sensitive to the charge transport mechanism peculiarities than the average transport characteristics, and therefore may provide additional information about the conduction physics [17-19]. If we refrain from the discussion of the quantum fluctuations at extremely high frequencies, two basic frequency ranges have to be distinguished. At very low frequencies, one can expect the $1 / f$-type noise that is observed experimentally in a wide variety of conductors; see, for example, [17]. In most cases the noise scales approximately in accordance with the phenomenological Hooge formula $[17,20]$. For a $2 \mathrm{D}$ conductor, this formula can be presented as

$$
\frac{S_{I}(f)}{\langle I\rangle^{2}}=\frac{a^{2}}{L W} \frac{C(f)}{f},
$$

1 In the opposite limit of thermal noise, the broadband current fluctuations are described by the fluctuation-dissipation theorem and hence do not provide any information not already available from average transport characteristics. 
where $S_{I}(f)$ is the current spectral density, $L$ is the length of the conductor (along the current flow) and $C(f)$ is either a dimensionless constant or a weak function of the observation frequency $f$. In particular, many studies [17] have found that $C(f) / f \propto 1 / f^{p}$, where $p$ is typically between 1 and 2. For the particular case of hopping conduction, two major theories of $1 / f$ noise have been suggested, based, respectively, on 'carrier number' fluctuations [21-23] and 'mobility' fluctuations [24, 25] as possible origins of the noise. Unfortunately, both theories have been developed for the case of substantially nonvanishing temperatures, for which an accurate numerical study of noise is difficult even with currently available advanced simulation algorithms and supercomputer resources.

At relatively high frequencies, the noise spectral density is a very slow (practically constant) function of $f$, and may be considered as a mixture of thermal fluctuations and shot noise. In the most interesting case of sufficiently low temperatures, the thermal fluctuations are negligible, and the broadband fluctuations are completely due to electric charge discreteness (shot noise).

An emphasis of most recent studies has been on the suppression of the shot noise with respect to its Schottky value $2 e\langle I\rangle$. In particular, such suppression is a necessary condition for quasi-continuous charge transfer at relatively high frequencies $[4,5]$. If the current spectral density $S_{I}(f)$ is flat at $f \rightarrow 0$, it may be characterized by the Fano factor

$$
F \equiv \frac{S_{I}(0)}{2 e\langle I\rangle},
$$

so that the term 'shot noise suppression' means that $F<1$. Previous theoretical studies of shot noise at hopping in artificial (space-ordered) 1D [7, 26] and (both space-ordered and random) 2D $[9,10]$ systems have shown that the shot noise may be, indeed, suppressed, obeying

$$
F=\left(\frac{L_{\mathrm{c}}}{L}\right)^{\alpha}, \quad L \gg L_{\mathrm{c}},
$$

where $L_{\mathrm{c}}$ is a scaling constant interpreted as the average percolation cluster length (i.e. the average distance separating critical hops [2,27]) and $\alpha$ is a positive exponent. In fact, at $T \rightarrow 0$ in the limit of negligible Coulomb interactions, our prior results [10] show that $L_{\mathrm{c}}$ obeys the law

$$
L_{\mathrm{c}}=J\left(\frac{E_{0}}{E}\right)^{\mu} a,
$$

where $J$ is a dimensionless constant of the order of 1 , and the value of the numerical exponent is $\mu=0.98 \pm 0.08$, consistent with the estimate $\mu \approx 0.91$ based on directed percolation theory [10, 27-29].

Considering a very long conductor, one might suspect that the electron motion in distant parts should not be correlated. This assumption immediately leads to $\alpha=1$ [19]. However, both analytical and numerical results $[7,26]$ show that at 1D hopping without Coulomb interaction, $\alpha$ may be as low as $1 / 2$. This nontrivial result may be interpreted as a consequence of an essentially infinite correlation length in 1D conductors, due to the on-site interaction of hopping electrons. Even more surprisingly, the exponent $\alpha$ may be substantially below 1 even in 2D conductors. For systems on a regular lattice, and without the Coulomb interaction, numerical modelling [9] yields $\alpha=0.85 \pm 0.02$. In our recent work [10], this finding has been confirmed for $2 \mathrm{D}$ hopping in conductors with completely random distribution of localized sites both in space and in energy. Our most accurate result was $\alpha=0.76 \pm 0.08$, i.e. significantly below 1.

It has not been immediately clear how the inclusion of Coulomb interaction effects might affect this result. For 1D hopping with increasing strength of the Coulomb interaction, 
numerical results [7] show $\alpha$ crossing over from nearly $1 / 2$ up to 1 . The similar behaviour might be expected for 2D hopping, because the long-range correlations, apparently responsible for the difference between $\alpha$ and 1, should be suppressed by Coulomb interaction effects, provided that the conductor length $L$ is larger than a certain crossover length determined by the interaction constant $\chi$. Unfortunately, recent experiments $[8,30,31]$ could not help in answering this question; while giving a reliable confirmation of the shot noise suppression in longer conductors, their accuracy is not sufficient to resolve a possible (relatively minor) deviation of $\alpha$ from 1 .

The resolution of the problem of shot noise suppression in long conductors has been the main motivation for the numerical experiment described in this paper. However, since the calculation of DC transport characteristics is computationally much less demanding than that of current noise, we have used this opportunity to obtain accurate values for the slow functions $A$ and $B$ for the same model of $2 \mathrm{D}$ hopping.

\section{Model}

We have studied broad $2 \mathrm{D}$ rectangular conductors $\left(W \gg L_{\mathrm{c}}\right)$ with 'open' boundary conditions on the interfaces with well-conducting electrodes $[9,10]$. The conductors are assumed to be 'fully frustrated', with a large number of localized sites randomly distributed over the conductor area. At the sites, the corresponding electron 'seed' eigenenergies $\varepsilon^{(0)}$ are also random, being uniformly distributed over a sufficiently broad energy band $2 B$, so that the $2 \mathrm{D}$ density of states $v_{0}$ is constant at all energies relevant for conduction.

The carriers are permitted to hop from any site $j$ to any other site $k$ with the rate

$$
\gamma_{j k}=\Gamma_{j k} \exp \left(-\frac{r_{j k}}{a}\right),
$$

where $r_{j k}$ is the site separation distance and $\Gamma_{j k}$ contains the energy dependence (see below). Such exponential dependence on the length of a hop is standard for virtually all theoretical studies of hopping ${ }^{2}$. Following our prior work [9, 10], we take equation (15) literally even at small distances $r_{j k} \sim a$. The energy dependence of $\Gamma_{j k}$ is given by the usual formula [10]

$$
\hbar \Gamma_{j k}\left(\Delta U_{j k}\right)=g \frac{\Delta U_{j k}}{1-\exp \left(-\Delta U_{j k} / k_{\mathrm{B}} T\right)},
$$

where $g$ is a dimensionless parameter which determines the $2 \mathrm{D}$ conductivity scale $\sigma_{0} \equiv g e^{2} / \hbar,^{3}$ while $\Delta U_{j k}$ is the difference of the total system energy before and after the hop from site $j$ to site $k$ :

$$
\Delta U_{j k} \equiv U_{j}-U_{k}+e \mathbf{E r}_{j k}
$$

Here $U$ is the total internal energy of the system, including the effects of Coulomb interaction:

$$
U \equiv \sum_{l}\left[n_{l} \varepsilon_{l}^{(0)}+\frac{e^{2}}{2 \kappa}\left(n_{l}-\frac{1}{2}\right) \sum_{l^{\prime} \neq l}\left(n_{l^{\prime}}-\frac{1}{2}\right) G\left(r_{l}, r_{l^{\prime}}\right)\right]
$$

where $n_{l}=0$ or 1 is the occupation number of the $l$ th localized site. (Similar to earlier studies [2,3] of the Coulomb effect on hopping, we keep the system electroneutral by adding

2 Notice that in contrast with some prior works, we do not include the factor 2 into the definition of the exponent. This difference should be kept in mind at the level of result comparison.

3 Parameter $g$ must be small $(g \ll 1)$ in order to keep coherent quantum effects (leading to weak localization and metal-to-insulator transition) negligible. 
a background charge of $e / 2$ to each site.) $G\left(r_{j}, r_{k}\right)$ is the electrostatic Green's function

$$
\begin{aligned}
G\left(r_{j}, r_{k}\right)= & \sum_{n=-\infty}^{\infty}\left[\frac{1}{\sqrt{\left(2 n L+x_{k}-x_{j}\right)^{2}+\left(y_{k}-y_{j}\right)^{2}}}\right. \\
& \left.-\frac{1}{\sqrt{\left(2 n L+x_{k}+x_{j}\right)^{2}+\left(y_{k}-y_{j}\right)^{2}}}\right],
\end{aligned}
$$

which includes the effect of image charges representing the screening effect of external electrodes modelled as ideally conducting semi-spaces.

For practical calculations, we do not need to evaluate $U$ from equation (18), because this equation may be used to rewrite equation (17) in the explicit form

$$
\begin{aligned}
\Delta U_{j k}=\varepsilon_{j}^{(0)}- & \varepsilon_{k}^{(0)}+e \mathbf{E r}_{j k}+\frac{e^{2}}{\kappa} \sum_{l \neq j}\left(n_{l}-\frac{1}{2}\right) G\left(r_{j}, r_{l}\right) \\
& -\frac{e^{2}}{\kappa} \sum_{l \neq k}\left(n_{l}-\frac{1}{2}\right) G\left(r_{k}, r_{l}\right)+\frac{e^{2}}{\kappa} G\left(r_{j}, r_{k}\right) .
\end{aligned}
$$

The numerical study has been carried out by using the classical Monte Carlo technique based on the algorithm suggested by Bakhvalov et al [32], which has become the de facto standard for single-electron tunneling simulations [33]. In most cases, the calculated variables are averaged over several (many) conductors with independent random distributions of localized sites in space and energy, but the same macroscopic parameters. The spectral density of current fluctuations is calculated using the advanced algorithm described in detail in [11].

\section{Results}

In order to classify the physical regimes of hopping behaviour, it is useful to note that our model has four relevant energy scales:

(i) $1 / \nu_{0} a^{2}$ describes the energy spectrum discreteness,

(ii) $e E a$ is the scale of the electric field strength,

(iii) $e^{2} / \kappa a=\chi / \nu_{0} a^{2}$ characterizes the Coulomb interaction strength, and

(iv) $k_{\mathrm{B}} T$ is the scale of thermal fluctuations.

Our primary interest is in transport, especially its dependence on the applied electric field $E$, so that instead of comparing $e E a$ with the other three energy scales, we prefer to speak about three characteristic values of electric field, which should be compared with the actual E:

$e a E_{T} \equiv k_{\mathrm{B}} T=\frac{T}{T_{0}} \times \frac{1}{v_{0} a^{2}}, \quad$ ea $E_{0} \equiv \frac{1}{v_{0} a^{2}}, \quad e a E_{\mathrm{c}} \equiv \frac{e^{2}}{\kappa a}=\chi \times \frac{1}{v_{0} a^{2}}$.

We are not interested in the case of extremely high temperatures, so that we will always assume that $T \ll T_{0}$, i.e. $E_{T} \ll E_{0}$. On the other hand, the relative position of points $E_{\mathrm{c}}$ and $E_{0}$ on the field axis is determined by the normalized parameter of the Coulomb interaction strength:

$$
E_{\mathrm{c}} / E_{0}=\chi \equiv e^{2} v_{0} a / \kappa
$$




\subsection{Coulomb gap}

In order to understand the peculiarities of Coulomb interaction effects in our model, we started with a calculation of the single-particle density of states for the case of $T=0$ and $E=0$. Indeed, in all the Coulomb gap analyses we are aware of, the electrostatic boundary effects have been ignored by assuming

$$
G\left(r_{j}, r_{k}\right)=\frac{1}{r_{j k}} .
$$

On the contrary, in our Green's function (19) the image charge contribution may be substantial, so it has been essential to understand how this contribution affects the Coulomb gap formation.

Following the Coulomb gap literature [2,3], we define the effective single-particle energy of an electron on site $j$ as

$$
\varepsilon_{j} \equiv \varepsilon_{j}^{(0)}+\frac{e^{2}}{\kappa} \sum_{l \neq j}\left(n_{l}-\frac{1}{2}\right) G\left(r_{j}, r_{l}\right) .
$$

Note that our basic equation (20) may be conveniently rewritten in terms of $\varepsilon_{j}$ :

$$
\Delta U_{j k}=\varepsilon_{j}-\varepsilon_{k}+e \mathbf{E r}_{j k}+\frac{e^{2}}{\kappa} G\left(r_{j}, r_{k}\right) .
$$

The calculations of the single-particle density of states in the ground state of a system (in which all $\Delta U_{j k}$ are negative) require its 'annealing'. In our case the annealing is facilitated by the fact that our model allows hopping between any pair of sites. This is why the natural relaxation of the conductor at $T=0$ and $E=0$ gave the results undistinguishable from those obtained after an explicit annealing procedure (see, for example, [34]). Since the Monte Carlo algorithm used is not slowed down when all the transition rates are very low, the relaxation could be simulated very quickly.

Figure 1 shows our typical results for the single-particle density of states. The soft Coulomb gap at sufficiently low energies is clearly visible. The effects of screening by the external electrodes are shown in figure 1(a). The data labelled 'screened' correspond to the full Green's function (19), which includes the electrostatic screening effects of the external electrodes, while the results for the simple approximation (23) are marked 'unscreened'. The results show that for conductors of sufficiently large size, screening has virtually no effect on the Coulomb gap formation. In this limit, the linear part of the $v(\varepsilon)$ dependence corresponds to equation (1) with the self-consistent equation result $c=2 / \pi \approx 0.64$ cited above.

Figure 1(b) shows the single-particle density of states for three different values of an important technical parameter, the half-bandwidth $B$ of the seed energy band. One can see that the value of $B$ does not affect the single-particle density of states well inside the Coulomb gap, but may influence the results at larger energies, so that $B$ should always be chosen properly in each particular case.

All the results presented below have been obtained for conductor size $L \times W$ and energy bandwidth $2 B$ so large that the effects of screening and finite number of states are negligible.

\subsection{DC transport characteristics}

Figure 2 shows our Monte Carlo results for the DC conductivity $\sigma$ as a function of temperature $T$ for two values of the Coulomb interaction strength parameter $\chi$. The results for $\chi=0$ coincide with those discussed in our previous work [10]. In particular, for sufficiently low temperatures ( $E_{T} \ll E_{0}$ ), the Monte Carlo data may be fitted by equation (4) using a simple power law in temperature $T$ to estimate the pre-exponential (model-dependent) function 

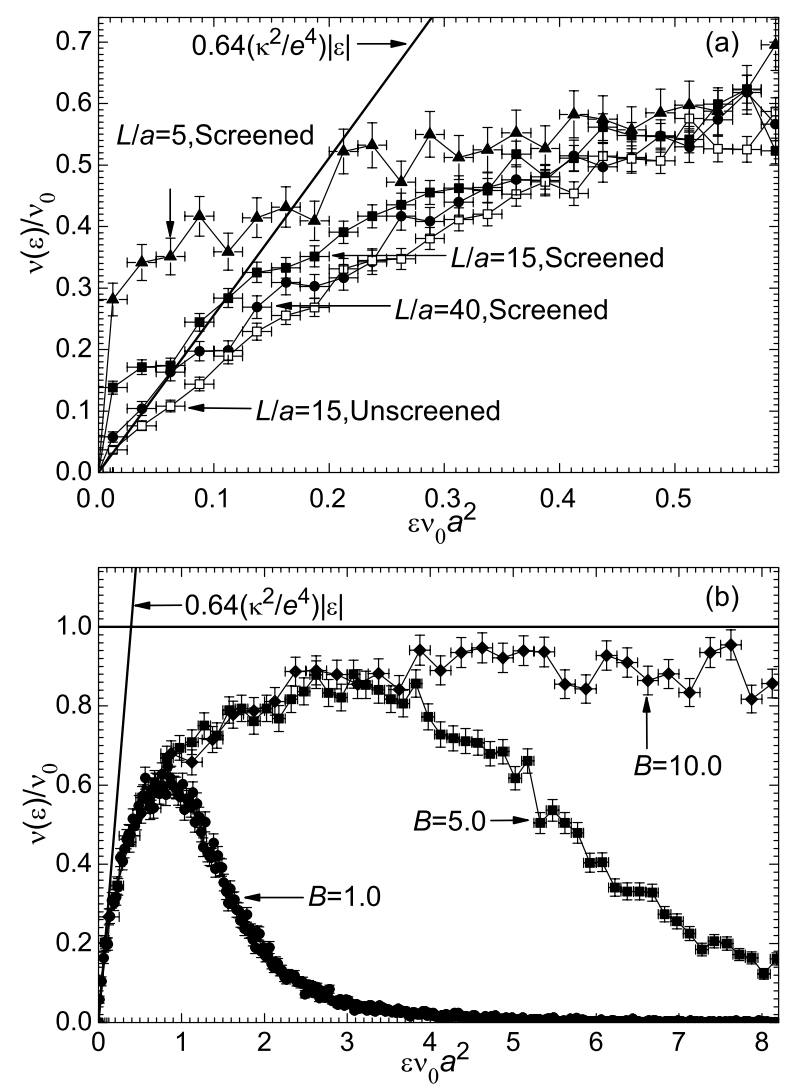

Figure 1. Single-particle density of states $v(\varepsilon) / \nu_{0}$ averaged over a large number of conductors at $\chi=0.5, T=0$ and $E=0$ for (a) several conductor lengths $L$ for large width $W / a=40$ at fixed half-bandwidth of the seed energy band $(B=1)$ with and without the screening due to electrostatic boundary effects, and for (b) several values of the half-bandwidth $B$ for sufficiently large conductors with screening. The straight lines correspond to equation (1) with $\alpha=2 / \pi \approx 0.64$. Curves are only guides for the eye.

$A(T, 0,0)=(23.4 \pm 1.3)\left(T / T_{0}\right)^{(0.68 \pm 0.04)}$ and a constant for $B(T, 0,0)=2.0 \pm 0.2$. (See [10] for a detailed discussion of this result.)

On the other hand, the results for $\chi=0.5$ show that in the case of substantial Coulomb interaction, the temperature dependence of conductance at low temperatures $\left(T / T_{0} \ll \chi^{3}\right)$ follows the Efros-Shklovskii variable-range hopping result (6). The best fitting gives $A(T, 0,0.5)=(10.7 \pm 1.3)\left(T / T_{0}\right)^{(1.02 \pm 0.12)}$ and $B(T, 0,0.5)=1.4 \pm 0.3$. This is in a reasonable agreement with the following values for $B$ (in our units): 3.1 following from an approximate analysis based on percolation theory [35], 4.8 found by evaluating an approximate integral over critical hops using a lattice model [36] and 2.9 obtained for a narrower range of temperatures using numerical (Monte Carlo) simulations on a uniform periodic lattice with randomly distributed energies [37]. (Unfortunately, the above values had no uncertainty reported.) The difference between our result and the reported values is probably due to the differences between details of the used models; see section 2 above.

For very high temperatures $\left(E_{T} \gtrsim E_{0}\right)$, the exponential temperature dependence of variable-range hopping theory cannot give a good description of the results, because in this case transport is dominated by very short hops with lengths of the order of the localization 


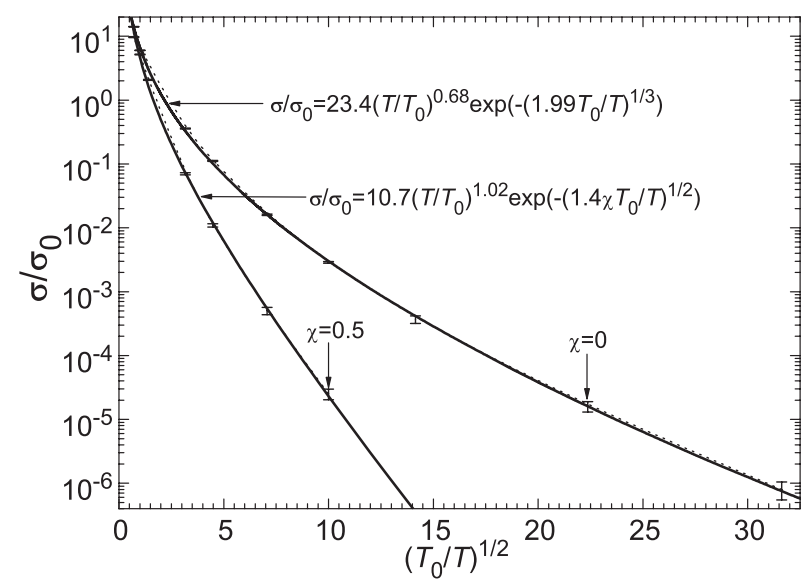

Figure 2. Linear DC conductivity $\sigma$ for negligible Coulomb interaction and finite Coulomb interaction as a function of temperature. Points show the Monte Carlo results which were obtained by the direct averaging of current calculated for a large number (from 20 to 96) of conductors with a random distribution of localized states, but the same macroscopic parameters. The sample size ranged from $20 a \times 14 a$ to $80 a \times 50 a$, depending on $\chi$ and $T$. Thin dashed lines are only guides for the eye, while the thick solid lines correspond to the best fits of the data by equations (4) and (6).

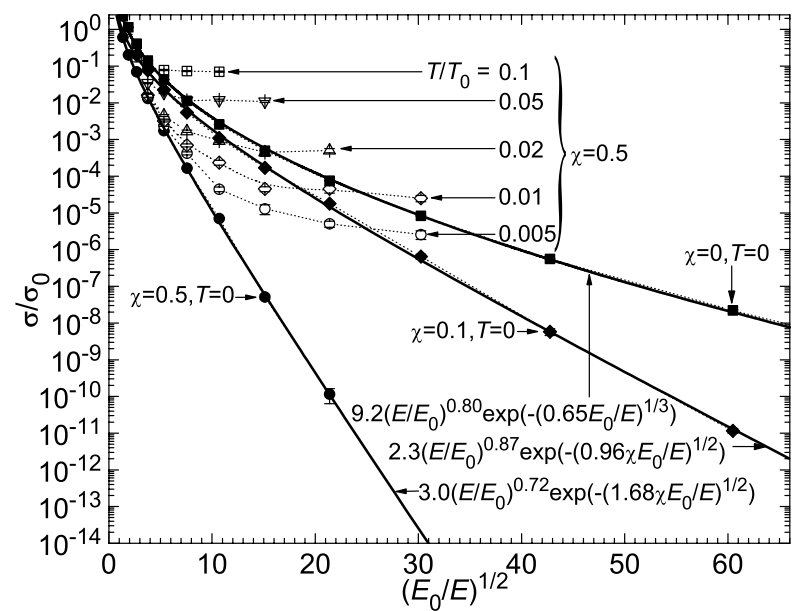

Figure 3. Nonlinear DC conductivity $\sigma$ as a function of electric field $E$ for several values of temperature $T$ and Coulomb interaction strength $\chi$. Points are Monte Carlo results averaged over a large number (from 20 to 96) of conductors of the same size (ranging from $20 \times 14 a^{2}$ to $800 \times 500 a^{2}$, depending on $\chi, T$ and $E$ ). Solid symbols show results for $T=0$, while open symbols correspond to $T \neq 0$. Thin dashed lines are only guides for the eye. Thick solid lines are the fits to the $T=0$ results using equations (8) and (10).

radius. However, even at these temperatures, Coulomb interaction effects lead to a drop in DC conductivity.

For higher electric fields $\left(E \gtrsim E_{T}\right)$, the DC current $\langle I\rangle$ increases faster than $E$, and therefore the DC conductivity, defined as $\sigma \equiv\langle I\rangle / E W$, becomes nonlinear and begins to increase with $E$; see figure 3. (More extensive data for the case of negligible Coulomb interaction, $\chi=0$, are shown in figure 2 of [10].) 


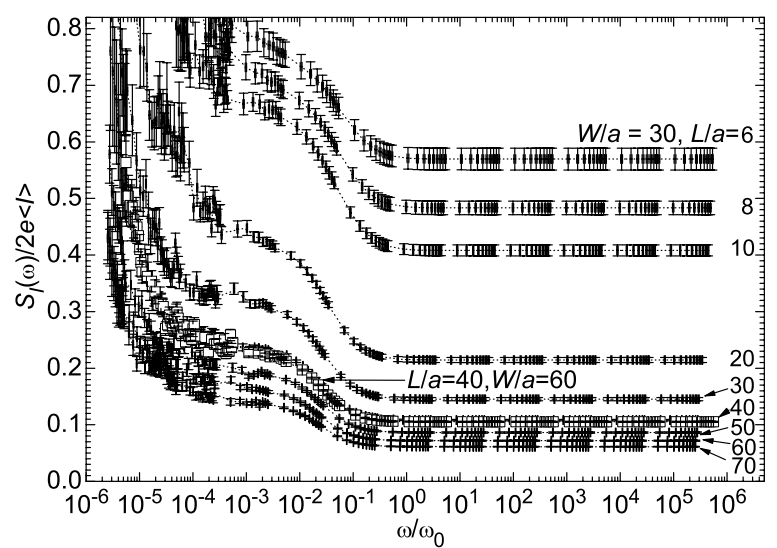

Figure 4. Spectral density $S_{I}(\omega)$ of current fluctuations at fixed Coulomb interaction strength $\chi=0.5$, as a function of observation frequency $\omega$ measured in units of $\omega_{0} \equiv g / \hbar v_{0} a^{2}$, for several values of conductor length $L$. Each point represents data averaged over 48 conductor samples at fixed parameters $\left(\chi=0.5, T=0\right.$ and $\left.E / E_{0}=0.07\right)$. Small points show results for $W / a=30$, while open squares are for $W / a=60$ (at $L / a=40$ ). Thin dashed lines are only guides for the eye.

For $T \rightarrow 0$, our results [10] for the case of negligible Coulomb interaction $(\chi=0)$ exhibit an exponential field dependence for not very high fields $\left(E_{T} \ll E \ll E_{0}\right)$ and may be fitted for the entire range of fields considered by equation (8) using (analogously to the low-field case) a simple power law in field $E$ to estimate the pre-exponential (model-dependent) function $A(0, E, 0)=(9.2 \pm 0.6)\left(E / E_{0}\right)^{(0.80 \pm 0.02)}$ along with constant $B(0, E, 0)=0.65 \pm 0.02$. (See [10] for a detailed discussion of this result.) The corresponding results for $\chi=0.1$ and $\chi=0.5$ show that increasing Coulomb interaction strength suppresses the nonlinear DC conductivity, just as in the low-field case. These results may be well fitted by equation (10) with $A(0, E, 0.1)=(2.3 \pm 0.6)\left(E / E_{0}\right)^{(0.87 \pm 0.07)}$ and $B(0, E, 0.1)=0.96 \pm 0.05$ for $\chi=0.1$ and $A(0, E, 0.5)=(3.0 \pm 0.4)\left(E / E_{0}\right)^{(0.72 \pm 0.07)}$ and $B(0, E, 0.5)=1.68 \pm 0.07$ for $\chi=0.5$. It is possible that any differences due to fitting reflect a (weak) systematic dependence on $\chi$.

The results for very high electric fields, $E \gtrsim E_{0}$ (near the localization limit), do not obey the variable-range hopping theory, due to very short hops of the order of the localization radius which dominate the transport in this case. Note, however, that even within this range the DC conductivity decreases with increasing Coulomb interaction strength.

To summarize our DC transport results, we see a very reasonable agreement with variablerange hopping theory within appropriate parameter ranges. Moreover, we believe that our supercomputer-enabled numerical modelling has given accurate parameters for the coefficients of these theories for our particular model.

\subsection{Current fluctuations}

3.3.1. $1 / f$ noise. Figure 4 shows typical results of our calculations of current noise at zero temperature, finite Coulomb interaction strength and fixed electric field, for several values of conductor length. Of particular note is that in sharp contrast with the negligible Coulomb interaction case [10], we do observe a $1 / f$-type noise at $f \rightarrow 0$. The frequency $f_{k}$ of the $1 / f$ noise 'knee' (the crossover from this noise to a quasi-flat spectral density) is relatively constant (or at most grows slowly with decreasing conductor length). This is what could be expected from the comparison of equations (11) and (13) if $\alpha \sim 1$. (For sufficiently large conductor 


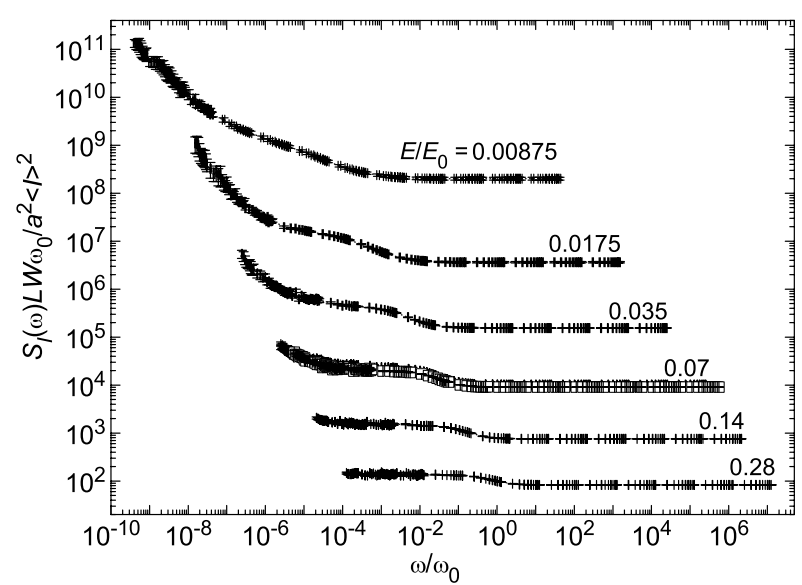

Figure 5. Spectral density $S_{I}(\omega)$ of current fluctuations at $T=0$ and $\chi=0.5$, normalized to the Hooge scaling factor $a^{2}\langle I\rangle^{2} / L W \omega_{0}$, as a function of observation frequency $\omega$ (measured in units of $\left.\omega_{0}\right)$ for several values of electric field. Each point represents data averaged over 48 conductor samples of the same size (ranging from $20 a \times 14 a$ to $120 a \times 60 a$, depending on $E$ ). Lines are only guides for the eye. For $E / E_{0}=0.07$, the results are plotted for a few conductor sizes, $50 a \times 30 a, 60 a \times 30 a$ and $70 a \times 30 a$ (small points) and 40a $\times 60 a$ (open squares). The results imply that the $1 / f$-type noise (in this normalization) is virtually size- and field-independent.

width $W,\langle I\rangle$ is proportional to $W$, so that $f_{k}$ should also be independent of $W$ as well, which is consistent with our results for different width in figure 4.)

In figure 5, the calculation results are plotted in the form allowing their straightforward comparison with the Hooge scaling $[17,20]$. Indeed in these coordinates, equation (11) with $C(f) / f \propto 1 / f^{p}$ would give a straight line dropping with slope $p$. We see that our data for $f \rightarrow 0$ are compatible with this formula, with $p \sim 1.3$. This value is close to the one calculated in [38] using a rather different model of hopping, apparently more adequate near the metal-insulator transition. The result is also compatible with recent experiments [39] which indicate an increase of $p$ from approximately 1 on the metallic side of such transition to above 1 on its dielectric side.

Unfortunately, more accurate determination of the noise spectral density $S_{I}(f)$ for sufficiently small frequencies and/or finite temperatures has been out of our reach, despite the use of advanced averaging algorithms [10] and unique supercomputer resources. As a result, at this stage we cannot compare our results with the existing theories of $1 / f$ noise at hopping [21-25, 40].

3.3.2. Fano factor and cluster length. If the low-frequency spectral density is constant (as it is for hopping at negligible Coulomb interaction [7, 9, 10]), it is naturally characterized by the Fano factor; see equation (12). In the presence of a $1 / f$-type noise, the definition of the Fano factor is less obvious. However, figures 4 and 5 show that the fluctuation spectrum has an exponentially broad plateau between the $1 / f$ noise knee and a crossover to another, high-frequency value. (The latter crossover at higher frequencies exists even in the absence of the Coulomb interaction; see [10] for a more detailed discussion.) The large length of these plateaus gives a motivation for the generalization of the Fano factor definition:

$$
F \equiv \frac{S_{I}\left(f_{p}\right)}{2 e\langle I\rangle},
$$




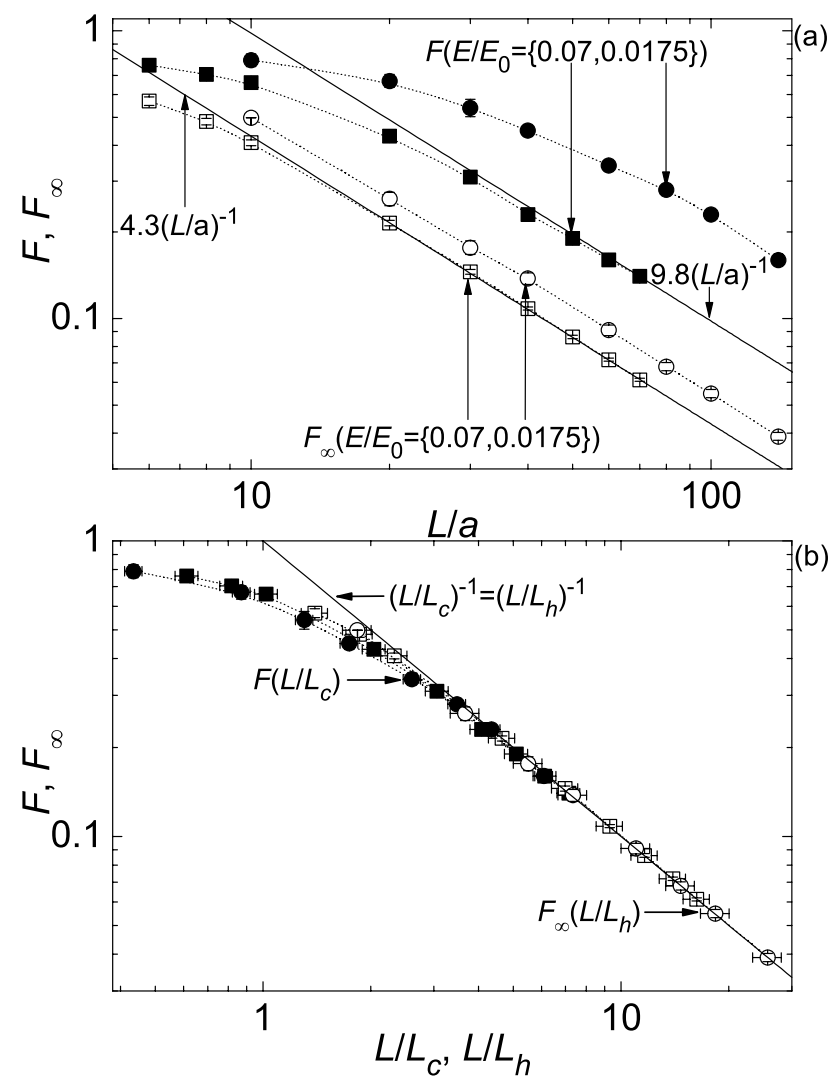

Figure 6. Average Fano factor $F$ and its high-frequency counterpart $F_{\infty}$ (equations (26) and (27), respectively) as functions of conductor length $L$ normalized to: (a) the localization length $a$, and (b) the scaling lengths $L_{\mathrm{c}}$ (for $F$ ) and $L_{\mathrm{h}}$ (for $F_{\infty}$ ) (see figure 7 below), for two values of applied field at $\chi=0.5, T=0$ and $W \gg L_{\mathrm{c}}$. Straight lines are the best fits to the data (using equations (13) and (28)), while dashed curves are only guides for the eye.

where $f_{p}$ is any frequency between the $1 / f$ noise knee and the high-frequency crossover. In addition, following [10], we may define a similar factor on the high-frequency plateau:

$$
F_{\infty} \equiv \frac{S_{I}(f \rightarrow \infty)}{2 e\langle I\rangle} .
$$

Figure 4 shows that neither of these factors depends on the sample width, at least for reasonably large $W$. Figure 6 shows the dependence of these factors on conductor length $L$. The results for $F$ in the case of substantial Coulomb interactions agree well ${ }^{4}$ with equation (13) with $\alpha \approx 1$, in contrast with the result $\alpha \neq 1$ for negligible interaction $[9,10]$. The results for the high-frequency counterpart $F_{\infty}$ agree well with the similar expression [10]

$$
F_{\infty}=\left(\frac{L_{\mathrm{h}}}{L}\right)^{\beta}, \quad L \gg L_{\mathrm{h}},
$$

\footnotetext{
4 In the opposite limit $L \ll L_{\mathrm{c}}$, when the number of hops necessary to cross the conductor is small, the Fano factor saturates at a level below 1. At negligible Coulomb interaction, the saturation level [10] is close to 0.7, i.e. the value consistent with the prior results for hopping through a parallel set of single sites, $\langle F\rangle=0.75$ [41], and two-site chains, $\langle F\rangle=0.707$ [42]. In the case of substantial Coulomb interaction, the Fano factor appears to saturate as well, though at a level somewhat above that for the negligible Coulomb interaction, possibly at $F \rightarrow 1$ when $\chi \rightarrow \infty$.
} 


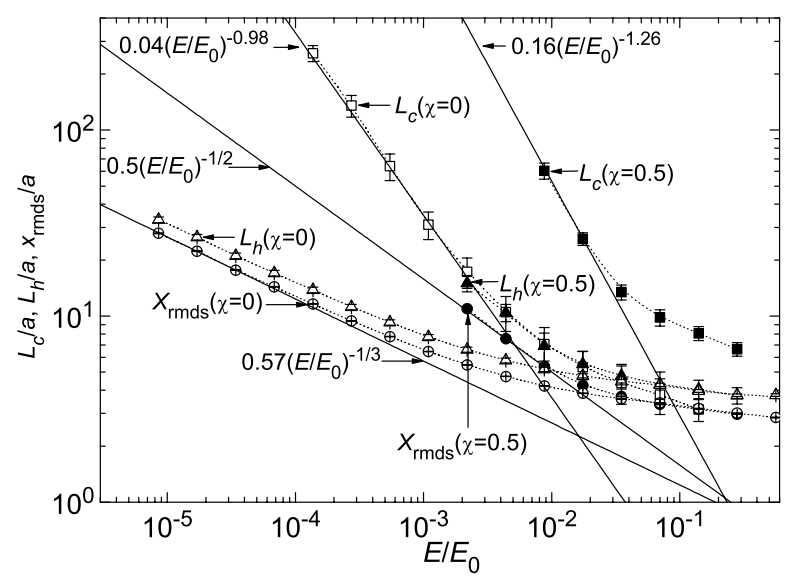

Figure 7. The values of parameters $L_{\mathrm{c}}$ and $L_{\mathrm{h}}$ giving the best fitting of shot noise results for equations (13) and (28), respectively, for sufficiently large conductors $\left(L, W \gg L_{\mathrm{c}}\right)$, as functions of electric field at $T=0$ for the cases of negligible ( $\chi=0$, open squares and triangles) and substantial $(\chi=0.5$, solid squares and triangles) Coulomb interaction. For comparison, circles show the results for the simple direction-weighted average hop length along the electric field direction (29). Dashed curves are only guides for the eye, while solid lines are the best fits using the variable-range hopping and percolation theory predictions (see the text).

where, within the accuracy of our calculations, $\beta=1$. This result is similar to that for negligible Coulomb interaction [10], and may be interpreted as a result of 'capacitive division' of the discrete increments of externally measured charge jumps resulting from single-electron hops through the system [43]. Figure 6(b) shows that both results can be collapsed onto a universal scaling curve by the introduction of certain length scales: $L_{\mathrm{c}}$ for $F$ and $L_{\mathrm{h}}$ for $F_{\infty}$.

In order to compare the corresponding length scales $\left(L_{\mathrm{c}}\right.$ and $\left.L_{\mathrm{h}}\right)$ with the proper measures of hop length, we have calculated the direction-weighted average [10] along the field direction

$$
x_{\mathrm{rmds}}^{2} \equiv \frac{\sum_{j, k} x_{j k}^{2}\left|H_{j k}-H_{k j}\right|}{\sum_{j, k}\left|H_{j k}-H_{k j}\right|},
$$

where $x_{j k} \equiv x_{k}-x_{j}=-x_{k j}$ is the component of the $j \rightarrow k$ hop length along the applied field direction, and $H_{j k}$ is the number of electrons making this hop during a certain time interval. For not too high fields $\left(E_{T} \ll E \ll E_{0}\right.$ ), the results in figure 7 for negligible Coulomb interaction are in good agreement with the variable-range hopping scaling described by equation (7), while for substantial Coulomb interaction they follow scaling similar to equation (9). In both cases, $L_{\mathrm{h}}$ and $x_{\text {rmds }}$ have a similar behaviour in the entire range of studied fields. (See [10] for a more detailed discussion on this result.) On the other hand, $L_{\mathrm{c}}$, as determined from equation (13), has a very different scaling, especially for lower fields ( $E \ll E_{0}$ ). Namely, at negligible Coulomb interaction, the results for $L_{\mathrm{c}}$ follow the law (14) with $J=0.04 \pm 0.01$ and $\mu=0.98 \pm 0.08$ [10] (see section 1.3), while in the case of substantial Coulomb interactions ( $\chi=0.5), L_{\mathrm{c}}$ also obeys equation (14), but with $J=0.16 \pm 0.02$ and $\mu=1.26 \pm 0.15$.

Following the analysis of [10], we may use the theory of directed percolation [27-29] to predict the following scaling:

$$
L_{\mathrm{c}} \propto\langle x\rangle\left(\frac{x_{\mathrm{c}}}{\left|\langle x\rangle-x_{\mathrm{c}}\right|}\right)^{\delta_{\|}},
$$


where $\langle x\rangle$ is approximately equal to $x_{\mathrm{rmds}}, x_{\mathrm{c}}$ is the critical hop length along the field, and the critical index $\delta_{\|}$should be close [29] to 1.73. Due to the exponential nature of the variablerange hopping scaling, $\left|\langle x\rangle-x_{\mathrm{c}}\right| \sim a$, and depending on the regime of hopping, we may use the corresponding field scaling of equations (7) or (9) (see figure 7) to arrive at equation (14) with either $\mu=\frac{1}{3}\left(1+\delta_{\|}\right) \approx 0.91$ or $\mu=\frac{1}{2}\left(1+\delta_{\|}\right) \approx 1.37$, respectively. These values are in a very reasonable agreement with our simulation results, thus confirming the interpretation of $L_{\mathrm{c}}$ as the average length of the directed percolation cluster.

In extremely high fields ( $E \gtrsim E_{0}$ ), the length scales $L_{\mathrm{c}}$ and $x_{\text {rmds }}$ become comparable to one another and both approach the localization radius $a$.

\section{Discussion}

To summarize, we have carried out numerical simulations of 2D hopping within a broad range of temperature, electric field and Coulomb interaction strength. For average (DC) transport characteristics, our results are in general agreement with the variable-range hopping theories, except for the (model-dependent) cases of 'ultra-high' electric field and/or temperature, where the hopping length becomes of the order of the localization radius.

For the spectrum of current fluctuations, our results are more significant. First, for the case of significant Coulomb interaction we have obtained a reliable evidence of $1 / f$-like fluctuations, approximately obeying the Hooge scaling (11), even at $T \rightarrow 0$. In hindsight, this result does not seem too surprising. Due to the presence of Coulomb interaction, random motion of the electrons during hopping transport generates a time-and space-varying Coulomb field, with a quasi-white spectrum, even at $T=0$. The effect of such a randomly changing field on localized electrons aside from the hopping clusters should be qualitatively similar to that of thermal fluctuations that may lead to the $1 / f$ noise, for example following one of the scenarios described in [21-25]. Recent experiments for hopping in quasi-3D samples [44, 45], showing a very slow change of $1 / f$ noise intensity at $T \rightarrow 0$, seem qualitatively compatible with this interpretation.

Our second important result is that in the presence of significant Coulomb interaction, the quasi-white noise above the $1 / f$ noise knee is suppressed according to the scaling law (13) with $\alpha=1$ (within the accuracy of our numerical experiment). This result is consistent with the simple addition of mutually independent noise voltages generated by (conductor) sample sections connected in series, and hence with the existence of a finite correlation length. On the other hand, the results [10] for negligible Coulomb interactions give $\alpha=0.76 \pm 0.08<1$, and are inconsistent with the existence of such a length, at least on the scale of $L_{\mathrm{c}}$. However, in both cases the constant $L_{\mathrm{c}}$, participating in the scaling law (13), may be interpreted as the length between the critical hops, i.e. the directed percolation cluster length.

\section{Acknowledgments}

Fruitful discussions with A Efros, S Kogan, A Korotkov, V Kozub, M Pollak, C Reichhart, B Shklovskii and D Tsigankov are gratefully acknowledged. The work was supported in part by the Engineering Physics Program of the Office of Basic Energy Sciences at the US Department of Energy, and by the Semiconductor Research Corporation. We also acknowledge the use of the following supercomputer resources: SBU's cluster Njal (purchase and installation funded by the US DoD's DURINT program), Oak Ridge National Laboratory's IBM SP computer Eagle (funded by the Department of Energy's Office of Science and Energy Efficiency program), and also IBM SP system Tempest at Maui High Performance Computing Center and IBM SP system Habu at NAVO Shared Resource Center (computer time granted by DOD's High Performance Computing Modernization Program). 


\section{References}

[1] Mott N F and Davies J H 1979 Electronic Properties of Non-Crystalline Materials 2nd edn (Oxford: Oxford University Press)

Mott N F 1993 Conduction in Non-Crystalline Materials 2nd edn (Oxford: Clarendon)

[2] Shklovskii B I and Efros A L 1984 Electronic Properties of Doped Semiconductors (Berlin: Springer)

[3] Efros A L and Pollak M (ed) 1991 Hopping Transport in Solids (Amsterdam: Elsevier)

[4] Averin D V and Likharev K K 1991 Mesoscopic Phenomena in Solids ed B Altshuler et al (Amsterdam: Elsevier) p 173

[5] Matsuoka K A and Likharev K K 1998 Phys. Rev. B 5715613

[6] Likharev K K 1999 Proc. IEEE 87606

[7] Korotkov A N and Likharev K K 2000 Phys. Rev. B 6115975

[8] Kuznetsov V V, Mendez E E, Zuo X, Snider G L and Croke E T 2000 Phys. Rev. Lett. 85397

[9] Sverdlov V A, Korotkov A N and Likharev K K 2001 Phys. Rev. B 63 081302(R)

[10] Kinkhabwala Y A, Sverdlov V A, Korotkov A N and Likharev K K 2006 J. Phys.: Condens. Matter 181999 (Kinkhabwala Y A, Sverdlov V A, Korotkov A N and Likharev K K 2003 Preprint cond-mat/0302445)

[11] Sverdlov V A, Kinkhabwala Y A and Korotkov A N 2005 Preprint cond-mat/0504208

[12] Shklovskii B I 1972 Fiz. Tekh. Poluprov. 62335

Shklovskii B I 1973 Sov. Phys._Semicond. 61964

[13] Apsley N and Hughes H P 1974 Phil. Mag. 30963 Apsley N and Hughes H P 1975 Phil. Mag. 311327

[14] Pollack M and Riess I 1976 J. Phys. C: Solid State Phys. 92339

[15] Rentzsch R, Shlimak I S and Berger H 1979 Phys. Status Solidi a 54487

[16] van der Meer M, Schuchardt R and Keiper R 1982 Phys. Status Solidi b 110571

[17] Kogan Sh 1996 Electronic Noise and Fluctuations in Solids (Cambridge: Cambridge University Press)

[18] de Jong M J M and Beenakker C W J 1997 Mesoscopic Electron Transport (NATO ASI vol 345) ed L L Sohn, L P Kouwenhoven and G Schön (Dordrecht: Kluwer) p 225

[19] Blanter Ya M and Buttiker M 2000 Phys. Rep. 3362

[20] Hooge F N 1969 Phys. Lett. A 29139

[21] Shklovskii B I 1980 Solid State Commun. 33273 Kogan Sh M and Shklovskii B I 1981 Sov. Phys.-Semicond. 15605

[22] Shklovskii B I 2003 Phys. Rev. B 67045201

[23] Shtengel K and Yu C C 2003 Phys. Rev. B 67165106

[24] Kozub V I 1996 Solid State Commun. 97843

[25] Kozub V I, Baranovskii S D and Shlimak I 1999 Solid State Commun. 113587

[26] Derrida B 1998 Phys. Rep. 30165

[27] Stauffer D and Aharony A 1994 Introduction to Percolation Theory rev 2nd edn (Philadelphia: Taylor and Francis)

[28] Obukhov S P 1980 Physica A 101145

[29] Essam J W, De'Bell K, Adler J and Bhatti F M 1986 Phys. Rev. B 331982

[30] Roshko S H, Safonov S S, Savchenko A K, Tribe W R and Linfield E H 2002 Physica E 12861

[31] Savchenko A K, Safonov S S, Roshko S H, Bagrets D A, Jouravlev O N, Nazarov Y V, Linfield E H and Ritchie D A 2004 Phys. Status Solidi b 241 26-32

[32] Bakhvalov N S, Kazacha G S, Likharev K K and Serdyukova S I 1989 Sov. Phys._JETP 68581

[33] Wasshuber C 2001 Computational Single-Electronics (Berlin: Springer) chapter 3

[34] Kaplan D M, Sverdlov V A and Likharev K K 2003 Phys. Rev. B 68045321

[35] Nguen V L 1984 Sov. Phys._Semicond. 18207

[36] Levin E I, Nguen V L, Shklovskii B I and Efros A L 1987 Sov. Phys._JETP 65842

[37] Tsigankov D N and Efros A L 2002 Phys. Rev. Lett. 88176602

[38] Reichhardt C and Olson Reichhardt C J 2004 Phys. Rev. Lett. 93176405

[39] Jaroszynski J, Popovic D and Klapwijk T M 2002 Phys. Rev. Lett. 89276401

[40] Kogan S 1998 Phys. Rev. B 579736

[41] Nazarov Yu V and Struben J J R 1996 Phys. Rev. B 5315466

[42] Kinkhabwala Y A and Korotkov A N 2000 Phys. Rev. B 62 R7727

[43] Averin D V and Likharev K K 1991 Mesoscopic Phenomena in Solids ed B L Altshuler, P A Lee and R A Webb (Amsterdam: Elsevier) p 173

[44] See the data for $\mathrm{Fe}_{3} \mathrm{O}_{4}$ in Shlimak I, Kraftmakher Y, Ussyshkin R and Zilberberg K 1995 Solid State Commun. 93829

[45] Raquet B, Coey J M D, Wirth S and von Molnar S 1999 Phys. Rev. B 5912435 